\title{
DIFFUSION OF POTASSIUM ON NICKEL
}

\author{
M. BLASZCZySZYNoWA aNd R. BLASZCZySzyN \\ Institute of Experimental Physics, University of Wroclaw \\ Cybulskiego 36, 50-205 Wroclaw, Poland
}

(Received June 24, 1991; in revised form September 9, 1991)

\begin{abstract}
The surface diffusion of a potassium dose corresponding to the average coverage $\bar{\Theta}_{\mathrm{K}}=1.5$ on nickel was studied using the field emission technique in the temperature range of 78-133 K. In general, under such conditions diffusion proceeds with the sharp moving boundary and the activation energy $Q$ from $0.16 \mathrm{eV}$ to $0.36 \mathrm{eV}$ dependently on the crystallograplic directions. Free boundary migration with the energy $Q<0.16 \mathrm{eV}$ is expected on the close-packed regions $\{111\}$ and $\{001\}$ already at liquid $N_{2}$ temperature. The results are discussed in relation to the atomic structure of the nickel substrate taking into account the interaction in the adsorption layer.
\end{abstract}

PACS numbers: $68.35 . \mathrm{Fx}, 79.70 .+\mathrm{g}, 79.90 .+\mathrm{b}$

\section{Introduction}

This work is a complement of experimental studies of the surface diflusion which were carried out for small potassium doses corresponding to $\bar{\Theta}_{\mathrm{K}}=0.02$ on clean nickel [1]. The present result concerns the surface diffusion of higher potassium doses giving the average coverage $\bar{\Theta}_{K}=1.5$ on clean nickel, and it was obtained by using the field emission microscopy (FEM) method. Studies of the surface diffusion of potassium on nickel are important in view of many problems such as the development of equilibrated overlayers, epitaxial growth, adsorption and desorption kinetics and catalytic processes. As to catalysis: (1) potassium is as an "electronic" promoter affecting the elementary steps of catalytic reaction and (2) the specific field emitter surface can be treated as a catalytic sample consisting of various crystallographic planes [2]: 


\section{Experimental}

The experiments were done, as previously described [1], in the glass sealed-off field emission microscope, equipped with a Ti getter, a Bayard-Alpert gauge and a potassium ampoule. During the measurements the field emission tube was immersed in liquid $\mathrm{N}_{2}$ and the pressure was below $10^{-11}$ torr $\left(10^{-9} \mathrm{~Pa}\right)$. The details of cleaning the $99.999 \% \mathrm{Ni}$ emitter have been given in the papers $[1,3]$.

The surface diffusion of the $\mathrm{K}-\mathrm{Ni}$ system was investigated by lateral deposition of a large dose of potassium at $78 \mathrm{~K}$ onto a thermally clean Ni tip surface (Fig. 1a) and determining the spreading times of the adsorbate as a function of temperature in the range of $99-133 \mathrm{~K}$. The angle between the direction of the potassium evaporation flux and the tip axis was about 90 degrees. Deposition of $\mathrm{K}$ and the monitoring of the diffusion was done at the field off. The magnitude of the $\mathrm{K}$ dose corresponded to the average coverage $\bar{\Theta}_{\mathrm{K}}=1.5$ after temperature equilibration of the adsorbate. The activation energy $Q$ for the diffusion was determined from Arrhenius-like plots for three crystallographic zones of the Ni surface (111)-(001), (110)-(001) and (111)-(110) as it can be seen in the stereographic projection of the fcc lattice (111)-oriented (Fig. 1b).

\section{Results}

According to the well-known relation

$$
\left\langle r^{2}\right\rangle=4 D t
$$

(where $\left\langle r^{2}\right\rangle$ stands for the mean square displacement of the moving sharp boundary within a time $t$ and $D$ the chemical diffusion coefficient) [4] plots of the distance $\Delta x(\propto r)$ versus the square root of the time $t$ are shown in Figs. 2, 3 and 5 for the zones (111)-(001), (110)-(001) and (111)-(110), respectively. The distance $\Delta x$ was determined from the field emission patterns (by means of the enlarger) in arbitrary units a.u. with the accuracy $\pm 1 / 2$ a.u. The distance $r$ on the tip surface can be found from the tip radius (about $7000 \AA$ ) and the known angular separation of the crystal faces identifiable from the symmetry of the field eimission microscopy patterns but it was not attempted to obtain a quantitative relation. Spreading rates in this case depend on the crystallographic zone line. For instance, the average rates at the substrate temperature $115 \mathrm{~K}$ are $111 \AA / \mathrm{s}, 37 \AA / \mathrm{s}$ and $5 \AA / \mathrm{s}$ for the zones (111)-(001), (011)-(001) and (111)-(011), respectively. Points in some plots $\Delta x$ versus $t^{1 / 2}$, denoted by letters, correspond to field emission patterns obtained after stepwise heating periods $t$, at the indicated temperatures $T$, and are shown in Figs. 1, 4 and 6 for the zones (111)-(001), (110)-(001) and (111)-(110), respectively.

\subsection{Diffusion along zone Ni(111)-(001)}

Figure 1c shows the field emission patterns for the clean nickel emitter after deposition of the potassium dose at $78 \mathrm{~K}$ corresponding to $\bar{\theta}_{\mathrm{K}}=1.5$. The arrow indicates the direction of the $\mathrm{K}$ deposition. One can see that the boundary of the original deposit at $78 \mathrm{~K}$ is highly anisotropic and the adsorbate covers some 
(a)

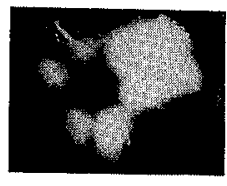

I K

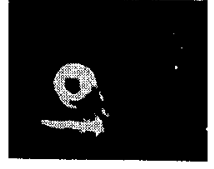

(c) $t=0$ "

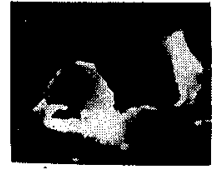

(g) $t=50^{\prime \prime}$

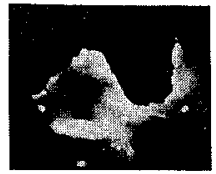

(k) $t=240^{\circ}$

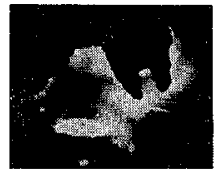

(o) $t=420^{\prime \prime}$

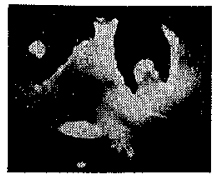

(t) $t=1320^{\prime \prime}$

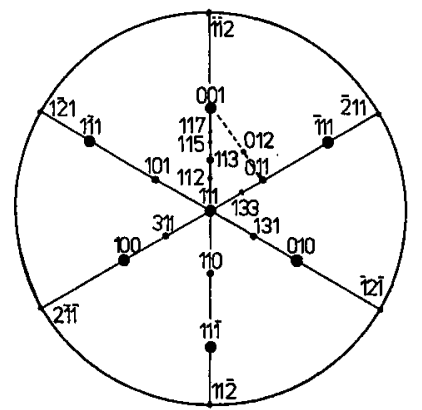

(b)

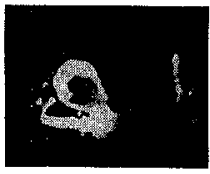

(e) $t=20^{\prime \prime}$

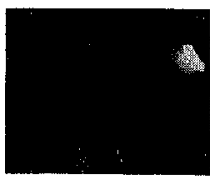

(i) $t=85^{\prime \prime}$
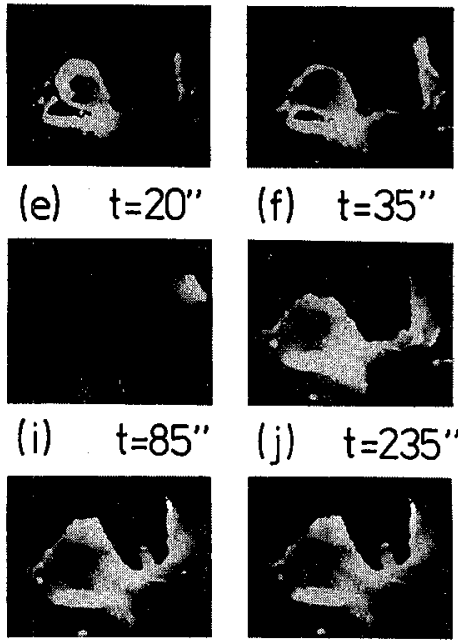

(f) $t=35^{\prime \prime}$

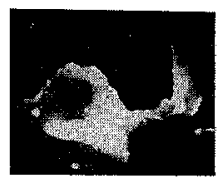

(h) $t=65^{\prime \prime}$

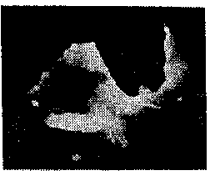

(l) $t=300^{\prime \prime}$

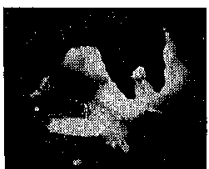

(m) $t=330^{\prime \prime}$
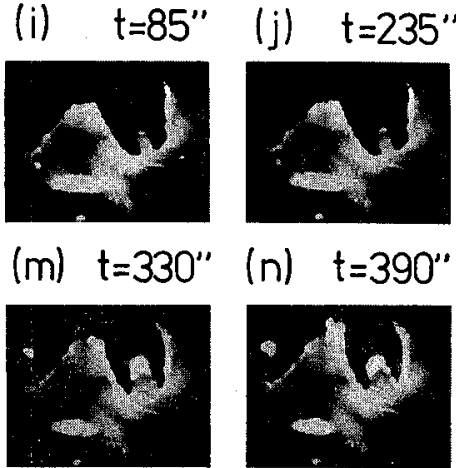

(n) $t=390^{\prime \prime}$

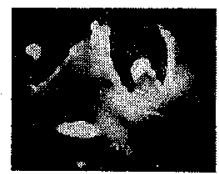

(p) $t=540^{\prime \prime}$

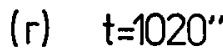

(s) $t=1260 "$
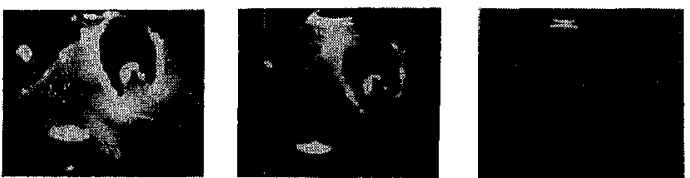

(v) $t=1900^{\prime \prime}(w) \quad t=730^{\prime \prime}$

Fig. 1. Field emission patterns, characteristic of: (a) clean nickel; (c) with initial potassium deposit at $78 \mathrm{~K}\left(\bar{\Theta}_{\mathrm{K}}=1.5\right)$; (d) $-(\mathrm{j})$ after heating at $98 \mathrm{~K}$ with the dominant diffusion along the zone (010)-(111); (k)-(r) after heating of the tip of (c) at $99 \mathrm{~K}$ with the dominant diffusion along zones $\{010\}-\{011\} ;(s)-(v)$ after heating of the tip of (c) at $99 \mathrm{~K}$ with the dominant diffusion around the (001) face; (w) after heating of the tip of (c) at $101 \mathrm{~K}$; $t$ represents the diffusion time; (b) stereographic projection of the fcc lattice (111)-oriented. 


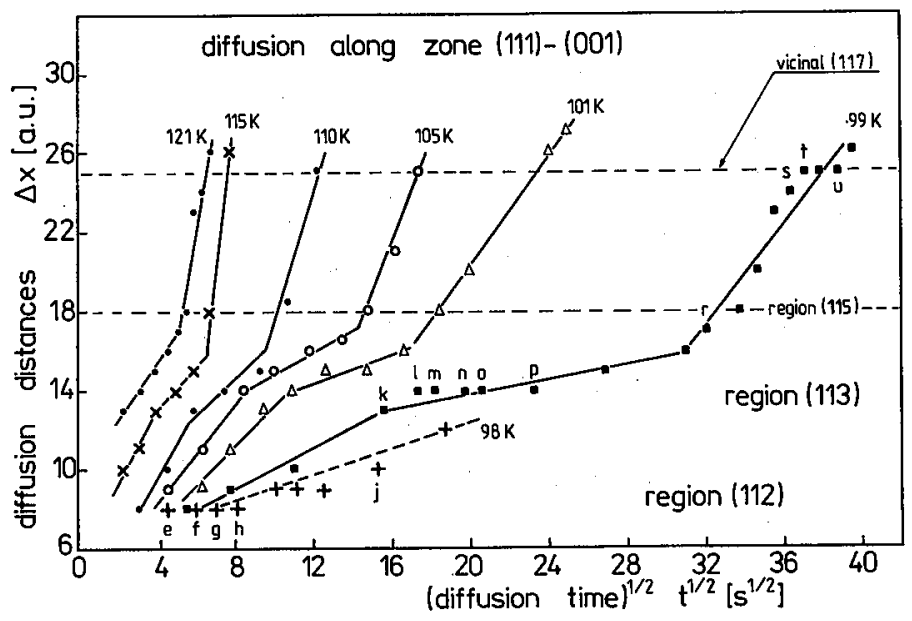

Fig. 2. Plots of average distance $\Delta x$ versus the square root of the spreading time $t^{1 / 2}$ for the zone $\mathrm{Ni}(111)-(001)$ at several temperatures. The horizontal dashed lines indicate the diffusion interval chosen for determining the activation energy. Points denoted by the letters correspond to the field emission patterns shown in Fig. 1.

regions on the shadow side of the emitter reaching the most closely packed $\mathrm{Ni}(111)$ plane via (131)-(111) zone. This indicates that the diffusion occurs on some regions during the deposition process at liquid nitrogen temperature. Typical stages in the progress of diffusion along the zone $\mathrm{Ni}(111)-(001)$ are shown in Figs. 1d-1v for the temperature range from $98 \mathrm{~K}$ to $101 \mathrm{~K}$, where $t$ is the heating time. Heating at these temperatures produces adsorbate spreading with a sharp boundary along the atomically rough surface zone. This trend is reproducible at higher temperatures.

The spreading rate of the sharp boundary is not uniform along this crystallographic zone. Plots of $\Delta x$ versus $t^{1 / 2}$ in Fig. 2 indicate that the boundary moves fastest in the stretch between the (113) and (001) planes and is distinctly moderated on some regions between the (112) and (113) planes and near the (001) plane. It can be also deduced from Figs. 1 and 2 that when the boundary motion in the vicinal (112) plane is moderate enhanced diffusion occurs along the zone (010)-(111) (Fig. 1e-1h). It is manifested by the increase of the emission from the (111) region in relation to the entire emitting surface. The emission from the (111) plane dominates completely after $t=85 \mathrm{~s}$ when the work function for this plane with potassium is to be minimum (Fig. 1i). Similarly, when the adsorbate reaches the vicinals of the (113) plane $(\Delta x=14$ a.u.) its motion seems to be stopped and simultaneously the difusion of the boundary proceeds along the zones (010)-(011) (Fig. $1 \mathrm{k}-1 \mathrm{p}$ ). The prolonged time of spreading leads to the arrival of the $\mathrm{K}$ atoms at the (001) plane (Figs $1 \mathrm{~s}-1 \mathrm{w}$ ). Figure $1 \mathrm{w}$ corresponds to the maximum emission of the plane when the plane shows a work function minimum similarly to the case of the $\{111\}$ planes. 


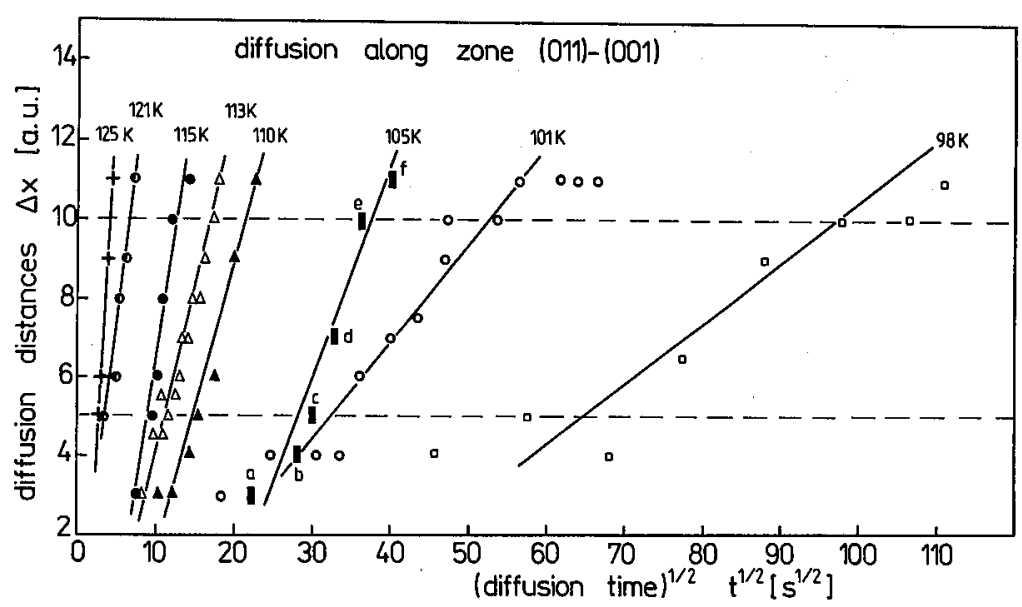

Fig. 3. Plots of average distance $\Delta x$ versus the square root of the spreading time $t^{1 / 2}$ for the zone $\mathrm{Ni}(011)-(001)$ at several temperatures. The horizontal dashed lines indicate the diffusion interval chosen for determining the activation energy. Points denoted by the letters correspond to the field emission patterns shown in Fig. 4.
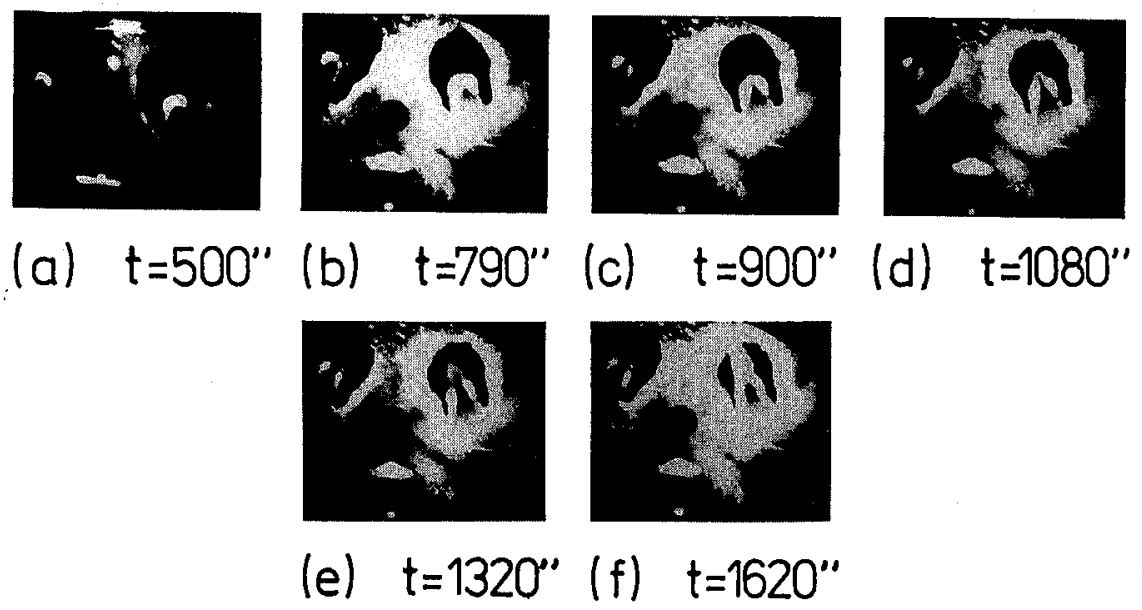

Fig. 4. (a)-(f) Nickel surface of Fig. 1c after heating time $t$ at $105 \mathrm{~K}$ with the dominant diffusion of $\mathrm{K}$ along the zone $\mathrm{Ni}(011)-(001)$.

Plots of $\ln t$ versus $1 / T$ for the zone (111)-(001) are shown in Fig. 7 . The time $t$ is the one found from the plots in Fig. 2 for the difference $\Delta x$ from 18 to 25 a.u. The respective activation energy of diflusion $Q_{1}$ equals $(0.16 \pm 0.02)$ $\mathrm{eV}$. Similar value of the $Q$ was determined for the entire diffusion region. Figure 2 shows that if the adsorbate is less available (at a lower temperature), $\mathrm{K}$ atoms first migrate boundary free on the close-packed $\{111\}$ and $\{001\}$ planes and then with 


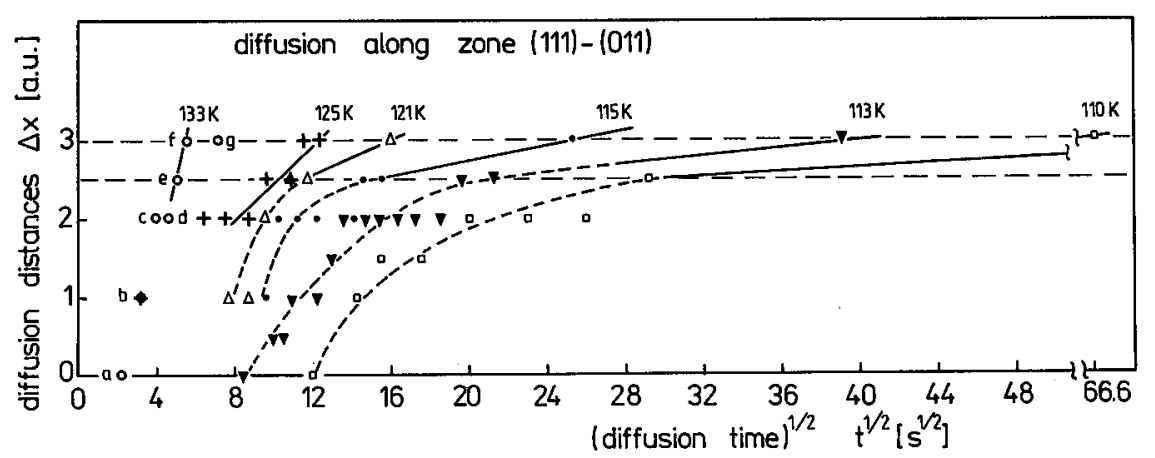

Fig. 5. Plots of average distance $\Delta x$ versus the square root of the spreading time $t^{1 / 2}$ for the zone $\mathrm{Ni}(111)-(011)$ at several temperatures. The horizontal dashed lines indicate the diffusion interval chosen for determining the activation energy. Points denoted by the letters correspond to the field emission patterns shown in Fig. 6.

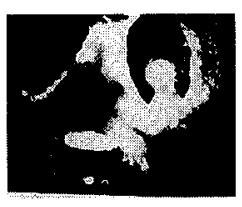

(a) $t=5^{\prime \prime}$

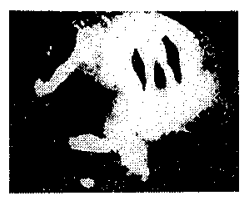

(e) $t=25^{\prime \prime}$

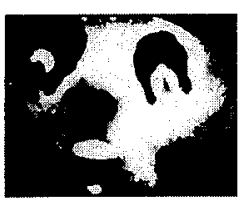

(b) $t=10 "$

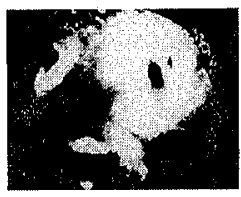

(f) $t=40^{\prime \prime}$
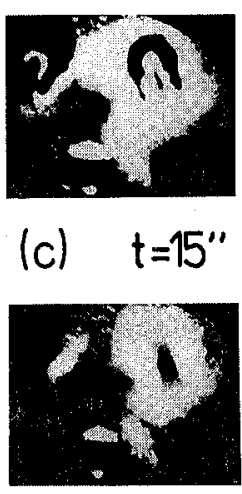

(g) $t=50^{\prime \prime}$

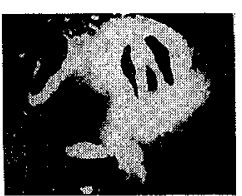

(d) $t=20^{\prime \prime}$

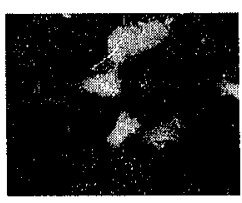

(h) $t=30^{\prime \prime}$

Fig. 6. (a)-(g) Nickel surface of Fig. 1c after heating time $t$ at $133 \mathrm{~K}$ with the dominant diffusion of $\mathrm{K}$ along the zone $\mathrm{Ni}(111)-(011)$; (g) state of the surface corresponding to the end point of diffusion; (h) "pseudoclean" pattern obtained after heating at $450 \mathrm{~K}$.

the sharp boundary on the atomically rough surface regions, e.g. over the edges of close-packed faces or terraces and the like. The diffusion on these close-packed planes proceeds so rapidly that activation energy measurements could not be made in the investigated temperature range of $98-133 \mathrm{~K}$.

\subsection{Diffusion along zone Ni(011)-(001)}

Plots of $\Delta x$ versus $t^{1 / 2}$ for the crystallographic zone (011)-(001) (Fig. 3) show that the spreading of potassium was uniform, i.e. the boundary motion obeys the relation (1) on all the zone. An Arrhenius plot for this zone is presented in 
Fig. 7. Respective activation energy $Q_{2}=(0.20 \pm 0.02) \mathrm{eV}$ was determined for the diffusion range shown in Fig. 3.

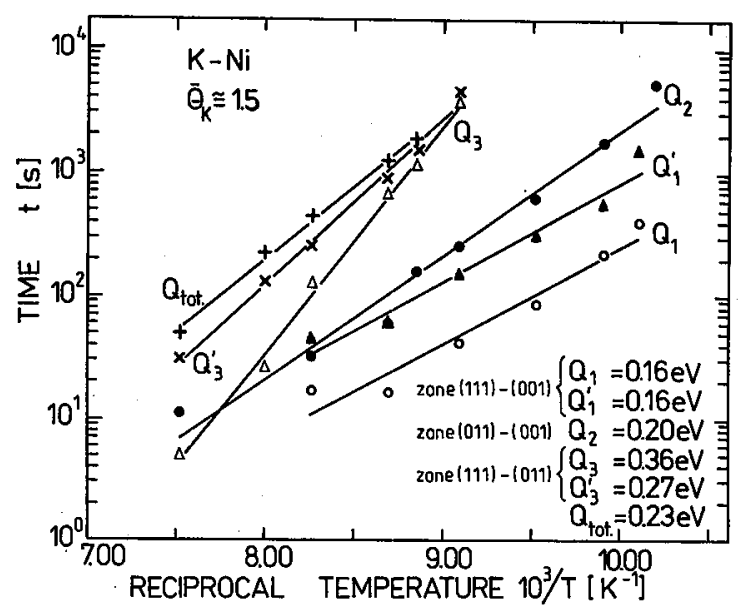

Fig. 7. Arrhenius plots for $\mathrm{K}$ diffusion along the $\mathrm{Ni}$ zones indicated and for equilibration of the whole original $K$ deposit, $Q_{t o t}$, corresponding to the field emitter pattern shown in Fig. 6.

\subsection{Diffusion along zone $\mathrm{Ni}(111)-(011)$}

Plots of $\Delta x$ versus $t^{1 / 2}$ for the zone (111)-(011) are shown in Fig. 5. The boundary motion does not obey the relation (1). This seems to indicate some variation in the chemical diffusion coefficient $D$. It depends on the ratio of the site number in the second or higher layer (physisorbed layer) to that in the first layer (chemisorbed layer) [4] and in this case it can alter because of: (1) the deposit (or its reservoir) is not infinite so that it becomes depleted; (2) the number of trap sites is not constant in this zone. Despite this we determine the diffusion activation energy for two diffusion ranges: from the initial deposit to the end point and for the small diffusion range corresponding to the difference of the $\Delta x$ from 2.5 to 3 a.u. (end point), see Fig. 5. The diffusion for these stages occurs with the activation energies of $Q_{3}=(0.27 \pm 0.02) \mathrm{eV}$ and $Q_{3}^{\prime}=(0.36 \pm 0.02) \mathrm{eV}$, respectively (Fig. 7). It is probably safer to accept the first value as the representative energy for the diffusion of potassium corresponding to $\bar{\Theta}_{\mathrm{K}}= \pm 1.5 \mathrm{eV}$ along the $\mathrm{Ni}(111)-(011)$ zone.

\section{Discussion}

In general, two categories of chemical diffusion are distinquished [5]: (1) for large initial deposits diffusion occurs with a sharp boundary and (2) if the initial deposit corresponds to the submonolayer coverage, boundary free migration 
is observed. The sharp boundary diffusion mechanism works for the diffusion of atoms in the upper layer to the edge of the covered region where they are trapped on the bare surface over which the migration barrier is high. The distinction can also arise owing to the effect of lateral interactions between adatoms [6].

TABLE

Summary of results on diffusion experiments.

\begin{tabular}{c|c|c|c|c|c}
\hline \hline $\begin{array}{c}\text { Diffusion } \\
\text { type }\end{array}$ & $\begin{array}{c}\text { Region } \\
\text { of surface }\end{array}$ & Temp. & $\begin{array}{c}\text { Average } \\
\text { potassium } \\
\text { coverage }\end{array}$ & $\begin{array}{c}\text { Activation } \\
\text { energy } \\
\text { of surface } \\
\text { diffusion } \\
Q[\mathrm{eV}]\end{array}$ & Ref. \\
\hline & $T[\mathrm{~K}]$ & $\bar{\Theta}_{\mathrm{K}}[-]$ & & \\
boundary & $(111)-(001)$ & $98-121$ & 1.5 & $0.16 \pm 0.02$ & this work \\
,-- & $(011)-(001)$ & $98-125$ & 1.5 & $0.20 \pm 0.02$ &,- \\
,-- & $(111)-(011)$ & $101-133$ & 1.5 & $0.27 \pm 0.02$ &,- \\
$-"$, & average & $98-133$ & 1.5 & $0.23 \pm 0.01$ &,- \\
boundary & $\{111\},\{001\}$ & $<78$ & $<1$ & $<0.16$ &,- \\
free & average & $211-254$ & 0.02 & $0.44 \pm 0.05$ & {$[1]$}
\end{tabular}

The results of diffusion experiments of potassium on nickel are summarized in the Table. In contradiction to the diffusion of $\mathrm{K}$ on $\mathrm{Ni}$ in the low coverage range $\left(\bar{\Theta}_{\mathrm{K}}<1\right)$ the diffusion of thick layer $\left(\bar{\Theta}_{\mathrm{K}}>1\right)$ proceeds with the sharp boundary like the other systems e.g. $[4,7,8]$. (The sharp boundary is observed when the jump distance of adatoms does not exceed the resolution of the microscope $20 \AA$.) The diffusion of a small $\mathrm{K}$ dose $\left(\bar{\Theta}_{\mathrm{K}}<1\right)$ over the entire nickel tip surface requires an activation energy value higher than for the large dose $\left(\bar{\Theta}_{\mathrm{K}}>\right)$ (see the Table). Thus the adatoms of the first layer are strongly bound to the substrate and immobile in the low temperature range. Adatoms at the coverages close to the submonolayer are less bound and more mobile at the lower temperatures. This diffusion picture is in agreement with the thermal desorption results $[9,2]$. The diffusion of large dose of $\mathrm{K}$ on the nickel tip proceeds with the smallest activation energy of 0.16 $\mathrm{eV}$ along the zone between $\{111\}-\{001\}$ planes which consist mainly of the close packed terraces of these planes. The roughest zone is the one between planes $\{111\}-\{110\}$ which is reflected in the relatively high diffusion activation energy. Although the diffusion of large dose proceeded mainly over the first potassium monolayer the substrate structure is not enough shielded by the first layer and the high diffusion anisotropy is observed.

It is interesting to discuss the diffusion of $\mathrm{K}$ atoms on the $\mathrm{Ni}(111)$ and (001) plancs. It is well-known that adsorption of alkali metals on metals distinctly reduces the work function of the substrate. The minimum of the work function of the system corresponds to the coverage $\bar{\Theta}_{\mathrm{K}}<1[9,2]$. For the close-packed planes the reduction is most marked. Figures $1 \mathrm{~d}, 1 \mathrm{i}$ and $1 \mathrm{w}$ demonstrate the dominating emission (the minimum work function) from the close-packed planes (111), (111) 
and (001) respectively. This means that the potassium coverage gradually increases on these planes. As the dimension of these planes are much greater than the resolution of the microscope one can expect the diffusion of $K$ atoms on these close-packed regions to proceed without sharp boundary, i.e. the $K$ atoms are believed to spread faster in the submonolayer coverage range than in the higher one with the activation energy $Q<0.16 \mathrm{eV}$. Let us note that in a macroscopic substrate experiment in the temperature range $40 \mathrm{~K}<T<150 \mathrm{~K}$, Hölal et al. have determined $Q=0.12 \mathrm{eV}$ for the $\mathrm{K} / \mathrm{Ni}(110)$ system [10].

The general rule that the diffusion barrier for adsorbates is approximately $10 \%$ to $40 \%$ of the adsorption barrier [5] leads to the conclusion that on the $\mathrm{Ni}(111)$ and (001) planes the diffusion barrier for $\mathrm{K}$ atoms should be higher for $\bar{\Theta}_{\mathrm{K}}<1$ than for the $\bar{\Theta}_{\mathrm{K}}>1$. (The desorption barrier distinctly decreases with increasing potassium coverage $[9,2]$.) However, on the atomically smooth surfaces the differences in the binding energies of $K$ atoms for various adsorption sites which correspond to the diffusion barrier $[11,6]$ may be greater in the second layer than in the submonolayers.

The work function and structure studies for $\mathrm{Na}, \mathrm{K}$ and $\mathrm{Cs}$ on the $\mathrm{Ni}(111)$, (100) planes confirmed the strong repulsive interaction between these species at submonolayer coverages $[9,2]$. According to the theoretical prediction under such conditions the diffuse boundary is expected [6]:

There might be also favorable conditions for other diffusion mechanism the one of soliton (collective) propagation mechanism [12,13]. This way of adsorbate spreading was proposed for the diffusion of $\mathrm{Ba}$ over the $\mathrm{Mo}(110)$ and $\mathrm{Li}$ on $\mathrm{W}(110)$ surfaces [14]. This mechanism is also taken into account for discussion of the field emission flicker noise cross-correlation results of a $\mathrm{K}$ submonolayer on the $\mathrm{W}(112)$ plane $[15,16]$, and $\mathrm{W}(110)[17]$.

\section{Conclusion}

It is interesting to note the following facts for a large initial potassium dose which gives the average coverage $\bar{\Theta}_{\mathrm{K}}=1.5$ on the Ni field emitter:

(1) $\mathrm{K}$ atoms migrate with the highest rate on the closest-packed regions of the $\mathrm{Ni}$ surface without a sharp diffusion boundary in the submonolayer coverage range with the activation energy $Q<0.16 \mathrm{eV}$. The diffusion proceeds at liquid nitrogen temperature.

(2) On the rough regions of the Ni sample $\mathrm{K}$ atoms migrate at temperature $T>98 \mathrm{~K}$ with a sharp anisotropic boundary. The activation energy increases in the sequence $Q_{1}<Q_{2}<Q_{3}$ for the zones (111)-(001), (011)-(001) and (111)-(011), respectively.

(3) The activation energy of the equilibration of this deposit $Q_{\text {tot }}=(0.23 \pm$ $0.01) \mathrm{eV}$ is considerably lower than the value of $Q=(0.44 \pm 0.05) \mathrm{eV}$ obtained for the diffusion of a small potassium dose $\left(\bar{\Theta}_{\mathrm{K}}=0.02\right)[1]$.

\section{Acknowledgements}

The help of Dr. S. Surma in the preparation of manuscript is gratefully acknowledged. 
This work was supported by the Polish Academy of Sciences, the Institute of Catalysis and Physico-Chemistry of Surfaces, Kraków; Project No.3.20 and the University of Wroclaw, Project No. 2252/W/IFD/91.

\section{References}

[1] M. Blaszczyszyn, Surf. Sci. 151, 351 (1985).

[2] H.P. Bonzel, Surf. Sci. Rep. 8, 43 (1987).

[3] M. Blaszczyszyn, R. Blaszczyszyn, R. Męclewski, A.J. Melmed, T.E. Madey, Surf. Sci. 131, 433 (1983).

[4] R. Gomer, in: Surface Mobilities on Solid Materials, Ed. Vu Thien Binh, Plenum Press, New York 1983, p. 7.

[5] R. Gomer, Field Emission and Field Ionization, Harvard University, Cambridge, M A, 1961.

[6] D.A. King, J. Vac. Sci. Technol. 17, 241 (1980).

[7] L.D. Schmidt, R. Gomer, J. Chem. Phys. 42, 3573 (1965).

[8] R. Męclewski, Acta Univ. Wratislau. Mat. Fiz. Astron. 147, 1 (X0) (1971) (in Polish).

[9] R.L. Gerlach, T.N. Rhodin, Surf. Sci. 19, 403 (1970); ibid. 17, 32 (1969).

[10] J Hölzl, to be published.

[11] G.L. Kellog, T.T. Tsong, P. Cowan, Surf. Sci. 70, 485 (1978).

[12] V.L. Pokrovsky, A.L. Talapov, Zh. Eksp. Teor. Fiz. 78, 269 (1980) (in Russian).

[13] I.F. Luksyutov, V.L. Pokrovsky, Pis'ma Zh. Eksp. Teor. Fiz. 33, 343 (1981) (in Russian).

[14] I.F. Luksyutov, A.G. Naumovets, S. Vedula, Solitons and Surface Diffusion, Ed. S.E. Trullinger, V.E. Zakharov, V.L. Pokrovsky, 12, 65 (1986).

[15] R. Blaszczyszyn, Ch. Kleint, Surf. Sci. 171, 615 (1986).

[16] J. Bęben, Ch. Kleint, A. Pawełek, Surf. Sci. 213, 451 (1989).

[17] R. Blaszczyszyn, Ch. Kleint, to be published. 